ated with other erythroid transcription factors binding in the intergenic $\gamma-\delta$ sequences are also active in human globin switching in both fetal and adult-type hematopoietic progenitors and erythroid cells. Inhibition of the activity of these complexes - for example, by using siRNAs directed against Ikaros or other components of other complexes - is a potential approach to reactivating human $\gamma$-globin expression.

The data in the Chakalova et al. article also indicate an inverse relationship between $\gamma$-and $\beta$-globin accumulation in adult erythroid cells (7). When there is more $\beta$-globin and HbA present, as found in the cells of patients doubly heterozygous for Corfu and $\beta$-thalassemia genes and patients heterozygous for the Corfu deletion, there are lower-than-expected levels of $\boldsymbol{\gamma}$-globin and $\mathrm{HbF}$ accumulation (7). These results suggest that in patients treated with butyrate or other $\mathrm{HbF}$-inducing agents, downregulating human $\beta$-globin expression may be a useful approach to further optimize $\mathrm{HbF}$ production.

In summary, upregulation of $\gamma$-globin and/or downregulation of $\alpha$-globin could reestablish normal globin balance between $\alpha$ - and non- $\alpha$-globin chains and avoid the excess $\alpha$-globin toxicity primarily responsible for anemia in human $\beta$-thalassemia. Achieving such a balance could ameliorate or even cure patients with these diseases. New insights into the mechanisms of globin regulation, such as those of Han et al. (3), may eventually lead to new, more rational treatments for patients with $\beta$-thalassemia.

Address correspondence to: Arthur Bank, Department of Medicine and Department of Genetics and Development, Columbia University College of Physicians and Surgeons, Armand Hammer Health Science Center, HHSC 16-1604, 701 West 168th Street, New York, New York 10032, USA. Phone: (212) 305-4186; Fax: (212) 923-2090; E-mail: ab13@columbia.edu.

1. Patrinos, G.P., et al. 2004. Multiple interactions between regulatory regions are required to stabilize an active chromatin hub. Genes Dev. 18:1495-1509.

2. Vakoc, C.R., et al. 2005. Proximity among distant regulatory elements at the $\beta$-globin locus requires GATA-1 and FOG-1. Mol. Cell. 17:453-462.

3. Han, A.-P., Fleming, M.D., and Chen, J.-J. 2005 Heme-regulated eIF2 $\alpha$ kinase modifies the phenotypic severity of murine models of erythropoietic protoporphyria and $\beta$-thalassemia. J. Clin. Invest. 115:1562-1570. doi:10.1172/JCI24141.
4. Kong, Y., et al. 2004. Loss of $\alpha$-hemoglobin-stabilizing protein impairs erythropoiesis and exacerbates $\beta$-thalassemia. J. Clin. Invest. 114:1457-1466. doi:10.1172/JCI200421982.

5. Kihm, A.J., et al. 2002. An abundant erythroid protein that stabilizes free $\alpha$-haemoglobin. Nature. 417:758-763.

6. Han, A.P., et al. 2001. Heme-regulated eIF $2 \alpha$ kinase (HRI) is required for translational regulation and survival of erythroid precursors in iron deficiency. EMBO J. 20:6909-6918.

7. Chakalova, L., et al. 2005. The Corfu $\delta \beta$ thalassemia deletion disrupts $\gamma$-globin gene silencing and reveals post-transcriptional regulation of $\mathrm{HbF}$ expression. Blood. 105:2154-2160.

8. O’Neill, D., Bornschlegel, K., Flamm, M., Castle, M., and Bank, A. 1991. A DNA-binding factor in adult hematopoietic cells interacts with a pyrimidinerich domain upstream from the human $\delta$-globin gene. Proc. Natl. Acad. Sci. U. S. A. 88:8953-8957.

9. O'Neill, D., et al. 1999. Tissue-specific and developmental stage-specific binding of a SWI/SNF complex associated with human fetal to adult globin switching. Proc. Natl. Acad. Sci. U. S. A. 96:349-354

10. Lopez, R.A., Schoetz, S., DeAngelis, K., O’Neill, D., and Bank, A. 2002. Multiple hematopoietic defects and delayed globin switching in Ikaros null mice. Proc. Natl. Acad. Sci. U. S. A. 99:602-607.

11. O'Neill, D., et al. 2000. An Ikaros-containing chromatin-remodeling complex in adult-type erythroid cells. Mol. Cell. Biol. 20:7572-7582.

12. Chen, J.S., Faller, D.V., and Spanjaard, R.A. 2003. Short-chain fatty acid inhibitors of histone deacetylases: promising anticancer therapeutics? Curr. Cancer Drug Targets. 3:219-236.

13. Davie, J.R. 2003. Inhibition of histone deacetylase activity by butyrate. J. Nutr. 133:2485S-2493S.

\title{
An oily, sustained counter-regulatory response to TB
}

\author{
Christopher L. Karp ${ }^{1}$ and Andrea M. Cooper ${ }^{2}$
}

\begin{abstract}
1Division of Molecular Immunology, Cincinnati Children's Hospital Medical Center and University of Cincinnati
\end{abstract} College of Medicine, Cincinnati, Ohio, USA. ${ }^{2 T r u d e a u ~ I n s t i t u t e ~ I n c ., ~ S a r a n a c ~ L a k e, ~ N e w ~ Y o r k, ~ U S A . ~}$

\begin{abstract}
Lipoxins are potent antiinflammatory lipid mediators that restrain and promote the resolution of a wide variety of inflammatory processes. Recent studies implicating deficient lipoxin production in the pathogenesis of diverse inflammatory diseases, along with numerous reports of the beneficial effects of lipoxin analog administration in animal models of inflammatory pathology, have suggested that harnessing the pleiotropic activities of the lipoxins is a strategy with considerable therapeutic promise. In this issue of the JCI, Bafica et al. address the other side of the coin, reporting that endogenous lipoxins compromise immune-mediated control of Mycobacterium tuberculosis infection in mice (see the related article beginning on page 1601). In addition to providing novel insight into the mechanisms that interfere with the development of protective immune responses to $M$. tuberculosis, the study raises the possibility that pharmacological inhibition of lipoxin synthesis may provide a method of augmenting inefficient immune responses in TB and other important chronic infectious diseases.
\end{abstract}

Nonstandard abbreviations used: LO, lipoxygenase; $\mathrm{LXA}_{4}$, lipoxin $\mathrm{A}_{4}$; NOS2, NO synthase 2.

Conflict of interest: The authors have declared that no conflict of interest exists.

Citation for this article: J. Clin. Invest. 115:1473-1476 (2005). doi:10.1172/JCI25353.
Maintenance of health is critically dependent upon the immune system's ability to generate a balanced response to a variety of threats, real or perceived. Inflammatory responses of insufficient vigor can allow uncontrolled pathogen replication, events central to the development of malaria, TB, and HIV, the top infectious killers in the world today. On the other hand, excessive or inappropriate inflammatory responses place an equally heavy burden on humanity, being key to the pathogenesis of diverse infectious (e.g., sepsis, fulminant viral hepatitis), autoimmune (e.g., inflammatory bowel disease, multiple sclerosis), allergic (e.g., asthma), genetic (e.g., cystic fibrosis), and degenerative (e.g., atherosclerosis) diseases. It is thus not surprising that there has been considerable experimental, theoretical, and therapeutic interest in the molecular mechanisms that restrain the intensity of inflammatory responses. In addition to the usual suspects, such as cytokines, receptors, intracellular signaling inhibitors, and specialized suppressor cells, endogenous antiinflammatory lipid mediators have recently been recognized as playing an important role. 


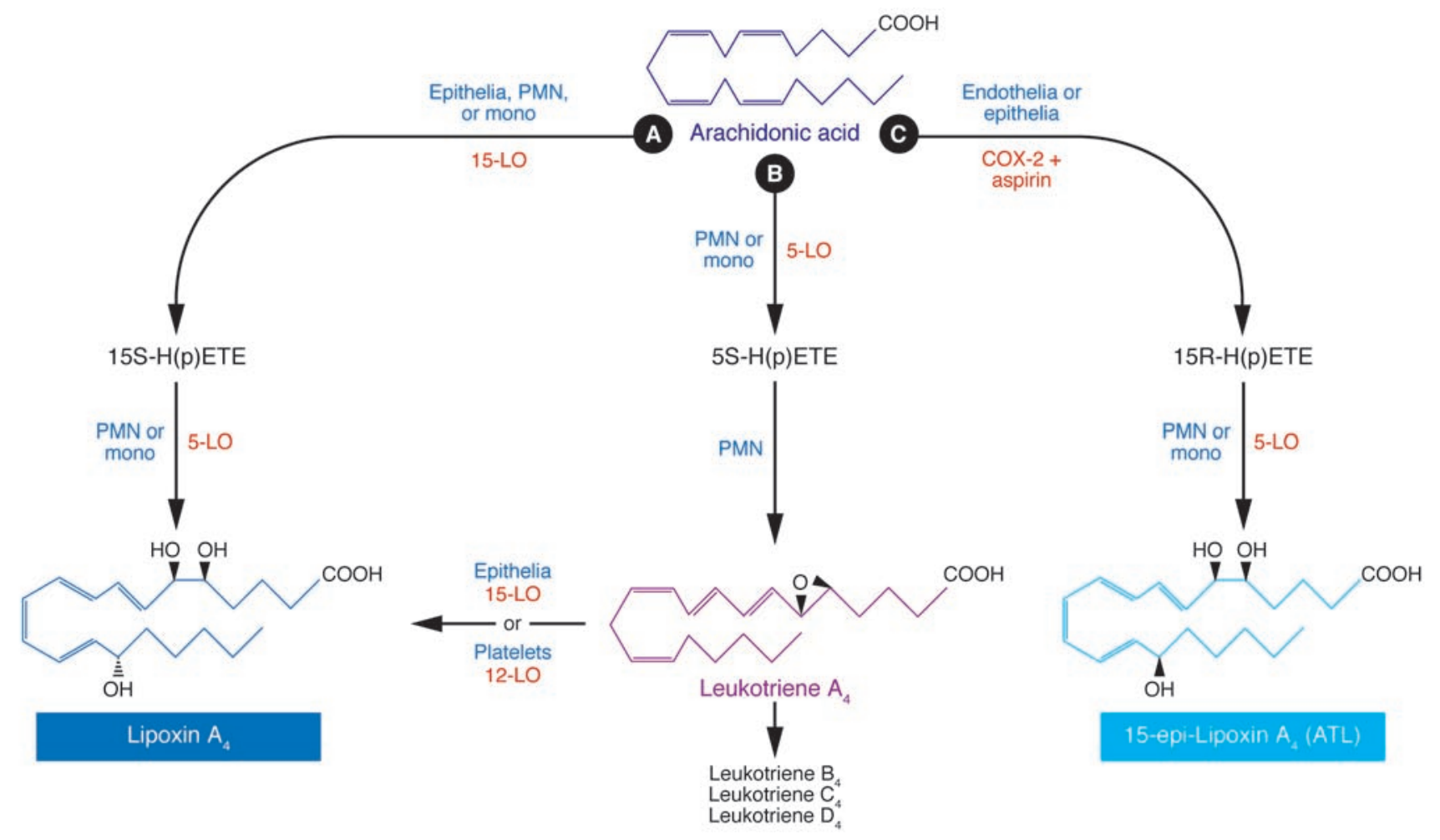

Figure 1

Lipoxin biosynthesis. There are at least 3 different biosynthetic pathways for lipoxin generation. All lead to the insertion of molecular oxygen at 2 sites in arachidonic acid by a variety of different enzymes that are generally segregated in different cell types and subject to regulation by cytokines and other inflammatory stimuli. In the first pathway (A), LXA $\mathrm{A}_{4}$ is generated through the action of 15-LO from airway epithelia or myeloid cells (neutrophils, monocyte/macrophages), which is followed by the action of 5-LO in myeloid cells. In the second pathway (B), LXA 4 is generated from leukotriene $\mathrm{A}_{4}\left(\mathrm{LTA}_{4}\right)$ (itself a product of 5-LO activity, and a leukotriene precursor) through the action of 15-LO or platelet-derived 12-LO. In the third pathway (C), arachidonic acid is converted to aspirin-triggered lipoxins (ATLs) such as 15-epi-LXA COX-2 in the presence of aspirin, which is followed by the action of 5-LO. The ATLs have similar activities but greater functional potency due to their relative resistance to metabolic inactivation. These pathways likely operate both independently and in a coregulated fashion in different tissues and biological situations. H(p)ETE, hydroperoxyeicosatetraenoic acid; mono, monocyte/macrophage; PMN, polymorphonuclear neutrophil.

\section{Counter-regulation by lipoxins and resolvins}

Lipoxins are trihydroxytetraene-containing arachidonic acid metabolites that are produced by at least 3 distinct lipoxygenase (LO) pathways, involving interactions among diverse cell types, including leukocytes, epithelia, endothelia, and platelets (Figure 1) (1). Our current understanding of the biologically important counter-regulatory activities of the lipoxins is largely due to the pioneering studies of the Serhan laboratory. Among in vitro activities, lipoxin $\mathrm{A}_{4}\left(\mathrm{LXA}_{4}\right)$ and/or its aspirin-triggered isomer, 15-epi-LXA $\mathrm{LA}_{4}$ (a) inhibit neutrophil chemotaxis, adherence, transmigration, and activation; (b) suppress epithelial cell and leukocyte production of diverse chemokines; (c) inhibit IL-12 production by DCs; (d) upregulate monocyte chemotaxis and ingestion of apoptotic neutrophils; and (e) suppress MMP production, while stimulating production of tissue inhibitors of MMPs (1-3). In vivo, lipoxins have been shown to have broad counter-regulatory properties, suppressing proinflammatory responses (preventing neutrophil-mediated damage; promoting the resolution of neutrophil-mediated inflammation), Th2-polarized responses (inhibiting inflammation and airway hyperresponsiveness in experimental asthma), and Th1 responses (suppressing immunopathology during infection with Toxoplasma gondii) alike $(1,4,5)$. This is a broad counter-regulatory profile, indeed. This profile, and the numerous reports of the beneficial effects of administering metabolically stable analogs in diverse mouse models of inflammatory pathology (1, 4, 6-10), suggest that harnessing the pleiotropic activities of the lipoxins is a strategy with considerable therapeutic promise. Lipoxin therapy may be particularly apt for diseases such as severe asthma and cystic fibrosis, where lipoxin deficiency has been implicated in disease pathogenesis (11-13).

It should also be noted that, in addition to those generated from arachidonic acid, immunoregulatory mediators are also generated from omega-3 fatty acids eicosapentaenoic acid and docosahexaenoic acid. Study of these latter mediators, named resolvins, is at an early stage, but immunologists (who have tended to pay little more than lip service to the biological importance of lipid mediators) should be aware that, like lipoxins, resolvins appear to have broad antiinflammatory, tissue protective, and catabatic properties $(14,15)$.

\section{Lipoxin-mediated control of the immune response to $M$. tuberculosis}

In $\mathrm{TB}$, bacterial persistence leads to a prolonged type 1 immune response in the lung that, while limiting bacterial growth, does not eliminate the highly inflammatory bacteria. This balance between bacte- 
ria and inflammation can be maintained for the life of the human host. However, breakdown leads to tissue damage, bacterial growth, and recrudescent disease. While the factors controlling this prolonged immune response have long been the subject of conjecture, the mechanisms responsible for counter-regulation during $\mathrm{TB}$ infection have remained obscure. The data implicating obvious candidates such as IL-10 and TGF- $\beta$, while intriguing, have remained associative in nature $(16,17)$.

In this context, the current report by Bafica et al. (18) demonstrating that lipoxins are key modulators of the immune response to $M$. tuberculosis is of considerable interest. Murine infection with M. tuberculosis led to significant, 5-LO-dependent production of $\mathrm{LXA}_{4}$. In turn, infected, 5-LOknockout mice had increased expression of the type 1 mediators IL-12, IFN- $\gamma$, and NO synthase 2 (NOS2) (but not TNF), along with better control of mycobacterial replication and enhanced survival. Notably, systemic treatment of 5-LO knockouts with a metabolically stable $\mathrm{LXA}_{4}$ analog reversed this enhanced control of mycobacterial replication. Such treatment also suppressed $M$. tuberculosis antigen-driven splenocyte production of IFN- $\gamma$ (but, again, not TNF). These data provide clear evidence of biologically important, lipoxin-mediated counter-regulation in experimental TB infection and provide what is believed to be only the second example of host gene ablation leading to better control of experimental infection with this pernicious pathogen, the first being the demonstration of the apparent regulatory role of the IL-27 receptor (19).

The lack of immunopathological consequences attending the upregulation of antimycobacterial immune responses in 5-LO-knockout mice is certainly a very welcome finding. This is not the case with T. gondii: despite improved control of parasite replication, 5-LO knockouts succumb rapidly to the encephalitic consequences of augmented inflammation during the early chronic phase of infection (5). As discussed by Bafica et al. (18), the different effect of 5-LO ablation on the course of these 2 infections likely relates to fundamental differences in the biology of the underlying pathogens. A vigorous Th1 response is necessary to contain infection with the rapidly replicating $T$. gondii, and such a response is induced in immunecompetent hosts. In contrast, the slowly replicating $M$. tuberculosis induces a less vigorous type 1 response (16). Removal of lipoxin-mediated counter-regulation leads to better control of pathogen replication in both infections. But this advantage is lost in toxoplasmosis, when the unleashing of the more potent antitoxoplasma response leads to fatal immunopathology.

Taken together, the data presented by Bafica et al. (18) suggest that pharmacological inhibition of lipoxin synthesis may allow for safe, therapeutic augmentation of the inefficient immune responses observed during TB infection. The presented control data are, perhaps, equally suggestive. $\mathrm{LXA}_{4}$ analog treatment of infected wild-type mice did not hamper the host response to $M$. tuberculosis. Similar findings have been presented in the context of experimental infection with T. gondii and Pseudomonas aeruginosa $(5,13)$. Thus, despite the important counter-regulatory role played by lipoxins, lipoxin analog treatment of lipoxin-sufficient animals does not appear to impair protective immune responses to pathogens, a finding with promise for the development of lipoxin analogs for the therapy of tissue inflammatory diseases.

For those used to the relatively direct relationship between gene and mediator among immune-associated proteins, the complexities of lipid mediator generation can be somewhat daunting. Ablation of 5-LO would be expected to ablate both $\mathrm{LXA}_{4}$ (and $\mathrm{LXB}_{4}$ ) and leukotriene production - antiinflammatory and proinflammatory mediators alike. This was indeed found in the current study (18). The overall effect of such ablation is, presumably, a reflection of the differing importance, kinetics, and cellular source of these mediators in the model in question. As for $\mathrm{LXA}_{4}$ itself, although the enzymatic events required for its generation from arachidonic acid are clear (oxygenation at the C5 and C15 positions), the specific LO enzymes that are necessary in any given context remain to be determined. In addition to redundancy in activity (e.g., both 15-LO and 12-LO can insert oxygen at C15) and celltype specificity, there are 7 LO genes in the mouse genome. The potential for each of these enzymes to affect the availability and activity of lipid mediators needs to be addressed by careful biochemical study. And even these LOs do not exhaust the possible sources of $\mathrm{LXA}_{4}$ generation during infection. Both T. gondii and P. aeruginosa encode enzymes with 15-LO activity $(20,21)$. Is it possible that a similar enzyme is lurking in the M.tuberculosis genome?
It is gratifying to see lipid mediators getting their due in immunopathogenesis. The current study should provide impetus for further mechanistic investigation of the role of counter-regulation by lipid mediators in TB. Important questions left open include whether the altered type 1 immunity observed in 5-LO-deficient mice is primarily a reflection of alterations in innate or adaptive immune responses, what enzymes and cells are critical for lipoxin production in this model, and what role the resolvins play in TB. There's much to be learned.

\section{Acknowledgments}

The authors thank N. Petasis for helpful discussions.

Address correspondence to: Christopher L. Karp, TCHRF 1566, 3333 Burnet Avenue, Cincinnati, Ohio 45229, USA. Phone: (513) 636-7608; Fax: (513) 636-5355; E-mail: chris.karp@chmcc.org.

1. Serhan, C.N. 2002. Lipoxins and aspirin-triggered 15-epi-lipoxin biosynthesis: an update and role in anti-inflammation and pro-resolution [review]. Prostaglandins Other Lipid Mediat. 68-69:433-455.

2. Aliberti, J., Hieny, S., Reis e Sousa, C., Serhan, C.N., and Sher, A. 2002. Lipoxin-mediated inhibition of IL-12 production by DCs: a mechanism for regulation of microbial immunity. Nat. Immunol. 3:76-82.

3. Sodin-Semrl, S., Taddeo, B., Tseng, D., Varga, J., and Fiore, S. 2000. Lipoxin A4 inhibits IL-1 betainduced IL-6, IL-8, and matrix metalloproteinase- 3 production in human synovial fibroblasts and enhances synthesis of tissue inhibitors of metalloproteinases. J. Immunol. 164:2660-2666.

4. Levy, B.D., et al. 2002. Multi-pronged inhibition of airway hyper-responsiveness and inflammation by lipoxin A(4). Nat. Med. 8:1018-1023.

5. Aliberti, J., Serhan, C., and Sher, A. 2002. Parasiteinduced lipoxin A4 is an endogenous regulator of IL-12 production and immunopathology in Toxoplasma gondii infection. J. Exp. Med. 196:1253-1262.

6. Clish, C.B., et al. 1999. Local and systemic delivery of a stable aspirin-triggered lipoxin prevents neutrophil recruitment in vivo. Proc. Natl. Acad. Sci. U. S. A. 96:8247-8252.

7. Gewirtz, A.T., et al. 2002. Lipoxin a 4 analogs attenuate induction of intestinal epithelial proinflammatory gene expression and reduce the severity of dextran sodium sulfate-induced colitis. J. Immunol. 168:5260-5267.

8. Leonard, M.O., et al. 2002. 15-Epi-16-(para-fluorophenoxy)-lipoxin $\mathrm{A}(4)$-methyl ester, a synthetic analogue of 15 -epi-lipoxin $\mathrm{A}(4)$, is protective in experimental ischemic acute renal failure. J. Am. Soc. Nephrol. 13:1657-1662.

9. Devchand, P.R., et al. 2005. A synthetic eicosanoid LX-mimetic unravels host-donor interactions in allogeneic BMT-induced GvHD to reveal an early protective role for host neutrophils. FASEB J. 19:203-210.

10. Pouliot, M., Clish, C.B., Petasis, N.A., Van Dyke, T.E., and Serhan, C.N. 2000. Lipoxin A(4) analogues inhibit leukocyte recruitment to Porphyromonas gingivalis: a role for cyclooxygenase- 2 and lipoxins in periodontal disease. Biochemistry. 39:4761-4768.

11. Sanak, M., et al. 2000. Aspirin-tolerant asthmat- 
ics generate more lipoxins than aspirin-intolerant asthmatics. Eur. Respir. J. 16:44-49.

12. Vachier, I., et al. 2005. Severe asthma is associated with a loss of LX4, an endogenous anti-inflammatory compound. J. Allergy Clin. Immunol. 115:55-60.

13. Karp, C.L., et al. 2004. Defective lipoxin-mediated anti-inflammatory activity in the cystic fibrosis airway. Nat. Immunol. 5:388-392.

14. Serhan, C.N., Gotlinger, K., Hong, S., and Arita, M. 2004. Resolvins, docosatrienes, and neuroprotectins, novel omega-3-derived mediators, and their aspirin-triggered endogenous epimers: an overview of their protective roles in catabasis. Prostaglandins
Other Lipid Mediat. 73:155-172.

15. Arita, M., et al. 2005. Stereochemical assignment, antiinflammatory properties, and receptor for the omega-3 lipid mediator resolvin E1. J. Exp. Med. 201:713-722

16. Flynn, J.L., and Chan, J. 2003. Immune evasion by Mycobacterium tuberculosis: living with the enemy. Curr. Opin. Immunol. 15:450-455.

17. North, R.J., and Jung, Y.J. 2004. Immunity to tuberculosis. Annu. Rev. Immunol. 22:599-623.

18. Bafica, A., et al. 2005. Host control of Mycobacterium tuberculosis is regulated by 5-lipoxygenase-dependent lipoxin production. J. Clin. Invest. 115:1601-1606.
doi:10.1172/JCI23949.

19. Pearl, J.E., et al. 2004. IL-27 signaling compromises control of bacterial growth in mycobacteria-infected mice. J. Immunol. 173:7490-7496.

20. Bannenberg, G.L., Aliberti, J., Hong, S., Sher, A., and Serhan, C. 2004. Exogenous pathogen and plant 15-lipoxygenase initiate endogenous lipoxin A4 biosynthesis. J. Exp. Med. 199:515-523.

21. Vance, R.E., Hong, S., Gronert, K., Serhan, C.N., and Mekalanos, J.J. 2004. The opportunistic pathogen Pseudomonas aeruginosa carries a secretable arachidonate 15-lipoxygenase. Proc. Natl. Acad. Sci. U. S. A. 101:2135-2139.

\title{
Cachexia in chronic kidney disease: a link to defective central nervous system control of appetite
}

\author{
William E. Mitch
}

Nephrology Division, Baylor College of Medicine, Houston, Texas, USA.

\begin{abstract}
Anorexia is one of several abnormalities characterizing chronic kidney disease (CKD) that cause cachexia, the loss of muscle and adipose stores. It has been attributed to mechanisms ranging from accumulation of toxic "middle molecules" to psychological problems. In this issue of the JCI, Cheung and coworkers used elegant techniques to demonstrate that CKD-associated anorexia is caused by defective hypothalamic regulation of appetite (see the related article beginning on page 1659). They attributed the defect to an alteration in the hypothalamus's response to leptin and inflammation. Since similar hypothalamic defects suppress appetite in inflammatory states and in cancer, it is possible that anorexia in several cachexia-inducing conditions results from a common set of hypothalamic abnormalities. The development of small molecules capable of preventing these regulatory abnormalities holds the promise of eliminating the contribution of anorexia to the development of cachexia.
\end{abstract}

The explosion of information emerging from several fields, including molecular and cellular biology and neuroscience, has helped elucidate specific mechanisms that regulate appetite. A major impetus for investigating why we eat is the increasing prevalence of obesity. However, there is a flip side: we also need to understand how catabolic conditions cause anorexia, which can contribute to cachexia - the loss of fat and protein stores. In chronic kidney dis-

Nonstandard abbreviations used: AGRP, agoutirelated peptide; AMPK, AMP-activated protein kinase; $\mathrm{CKD}$, chronic kidney disease; MC4-R, melanocortin receptor 4; $\alpha$-MSH, $\alpha$-melanocyte-stimulating hormone; NPY, neuropeptide Y; POMC, pro-opiomelanocortin; $\mathrm{PYY}_{3-36}$, peptide $\mathrm{YY}_{3-36}$.

Conflict of interest: The author has declared that no conflict of interest exists.

Citation for this article: J. Clin. Invest. 115:1476-1478 (2005). doi:10.1172/JCI25255. ease (CKD) there is evidence that the intake of calories, including protein, decreases as renal insufficiency advances (1). Although this is one reason for weight loss in CKD patients, metabolic abnormalities other than poor diet can also cause cachexia (2).

Early studies of the anorexia associated with CKD established a link between this condition and the circulation of "middle molecules" - compounds with molecular weights between 1.0 and $5.0 \mathrm{kDa}$. While the structures of these anorexigenic middle molecules are unknown, injection of a mixture of them into a normal rat's peritoneal cavity has been shown to decrease carbohydrate intake (3). Other potential causes of anorexia in CKD patients include a decreased ability to distinguish flavors (i.e., abnormal taste), stomach irritation caused by medicines such as iron compounds or phosphate binders, hemodynamic insta- bility as a result of exposure to antihypertensive medicines or hemodialysis, and a sensation of fullness during peritoneal dialysis, as well as psychological and economic factors.

\section{How does the central nervous} system integrate hunger and satiety? A study by Cheung and colleagues reported in this issue of the JCI (4) provides a quantum increase in our understanding of CKDassociated anorexia. Using animal models of CKD, the authors uncovered defects in the complex neuroendocrine pathways that regulate food intake. To appreciate the scope of their studies, a brief background is needed (see Figure 1). For over 50 years, it has been recognized that inhibitory signals proportional to body fat stores act to decrease food intake (5). The so-called long-term regulators of appetite - insulin and leptin - are produced in the pancreas and adipose cells, respectively. These regulators circulate at levels proportional to body fat and enter the brain in proportion to their plasma levels. In the hypothalamus, they influence neural pathways that integrate feeding and satiety as well as energy expenditure $(6,7)$. High levels of either leptin or insulin decrease food intake and increase energy expenditure, while low levels stimulate appetite and suppress energy expenditure. There are, however, important differences between the actions of insulin and leptin. A low leptin level increases fat accumulation, which ultimately raises cir- 